\title{
NEURL rs6584555 and CAND2 rs4642101 contribute to postoperative atrial fibrillation: a prospective study among Chinese population
}

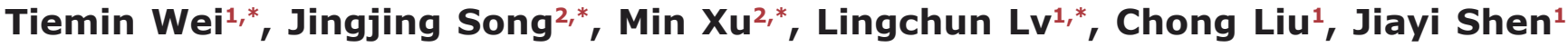 \\ and Ying Huang ${ }^{3}$ \\ ${ }^{1}$ Vasculocardiology Department, The Fifth Affiliated Hospital of Wenzhou Medical University, Affiliated Lishui Hospital of \\ Zhejiang University, The Central Hospital of Zhejiang Lishui, Lishui, Zhejiang, P.R. China \\ 2 Department of Radiology, The Fifth Affiliated Hospital of Wenzhou Medical University, Affiliated Lishui Hospital of Zhejiang \\ University, The Central Hospital of Zhejiang Lishui, Lishui, Zhejiang, P.R. China \\ ${ }^{3}$ Department of Cardiology, Sichuan Medical University, Sichuan, P.R. China \\ * These authors have contributed equally to this article \\ Correspondence to: Tiemin Wei, email: weitieminzj@126.com
}

Keywords: susceptibility; atrial fibrillation; coronary artery bypass graft surgery; SNP

Received: December 10,2015 Accepted: April 16, $2016 \quad$ Published: May 17, 2016

\section{ABSTRACT}

Postoperative atrial fibrillation (POAF) is a serious, common complication after coronary artery bypass grafting (CABG) surgery. Recently, 5 novel loci were identified to be associated with atrial fibrillation susceptibility using a combination of genotyping, eQTL mapping, and functional validation. In current study, we aim to evaluated the positive findings for POAF susceptibility after CABG among Chinese population, using a population-based, two-stage, nested case-control study with 1,400 patients. NEURL rs12415501 and CAND2 rs4642101 were significantly associated with POAF susceptibility after CABG among Chinese population in both stages. When pooled together, the ORs for each additional copy of minor allele was 1.29 (95\% CI: 1.13-1.48, $\left.P=1.7 \times 10^{-4}\right)$ for NEURL rs12415501, and 1.21 (95\% CI: 1.08-1.36, $P$ $=9.8 \times 10^{-4}$ ) for CAND2 rs4642101. Functional validation experiments found the AF risk allele of NEURL rs6584555 and CAND2 rs4642101 correlated with an increased expression of its corresponding genes $(P<0.001)$. In this independently collected cardiac surgery cohort, we replicated the previous findings, and 2 novel loci are independently associated with POAF risk in patients who undergo CABG surgery in Chinese population.

\section{INTRODUCTION}

Postoperative atrial fibrillation (POAF) is a serious yet common complication, also a known predictor of inhospital morbidity and short-term survival after coronary artery bypass grafting (CABG) surgery, occurring in $25 \%$ to $40 \%$ of patients [1-5]. However, pathophysiology of POAF is not fully disclosed [6-8]. General factors include older age, male gender, obesity, preexisting congestive heart failure, chronic renal failure, or COPD which are all risk factors for POAF [9-11]. Identification of patients who are at higher risk of POAF using genetic markers might be an important step for prevention of this type of operative complication and may provide important insight into the pathogenesis of atrial fibrillation (AF) and new therapeutic strategies for individual patients according to relative risk $[12,13]$.

During last 20 years, numerous AF associated mutations, candidate genes, and risk loci have been identified [14-19]; however, much of the heritability of AF remains unexplained [20]. Recently, Sinner et al [21] identified that 6 polymorphisms in 5 novel loci (NEURL rs12415501 and rs6584555, CAND2 rs4642101, GJA1 rs13216675, TBX5 rs10507248, and CUX2 rs6490029) were associated with atrial fibrillation susceptibility using a combination of genotyping, eQTL mapping, 
Table 1: Clinical characteristics of the study participants.

\begin{tabular}{|l|l|l|l|l|l|l|}
\hline & \multicolumn{2}{|l|}{ Stage I } & & Stage II \\
\hline Demographic & POAF $(\boldsymbol{N}=\mathbf{6 0 0})$ & $\begin{array}{l}\text { No POAF }(\boldsymbol{N}= \\
\mathbf{6 0 0})\end{array}$ & $\boldsymbol{P}$ value & POAF $(\boldsymbol{N}=\mathbf{8 0 0})$ & $\begin{array}{l}\text { No POAF } \\
\mathbf{8 0 0})\end{array}$ & $\boldsymbol{N}=$ \\
\hline Age, y & $65.5 \pm 5.2$ & $65.8 \pm 5.9$ & 0.350 & $62.7 \pm 4.9$ & $62.9 \pm 4.3$ & 0.386 \\
\hline Gender $(\%$ female) & $152(25.3 \%)$ & $149(24.9 \%)$ & 0.842 & $204(25.5 \%)$ & $201(25.1 \%)$ & 0.863 \\
\hline BMI, kg/m 2 & $22.9 \pm 2.3$ & $22.4 \pm 3.7$ & 0.005 & $23.1 \pm 3.2$ & $22.7 \pm 3.7$ & 0.021 \\
\hline Medical history & & & & & & \\
\hline Hypertension & $462(77.0 \%)$ & $455(75.9 \%)$ & 0.643 & $560(70.0 \%)$ & $567(70.9 \%)$ & 0.701 \\
\hline Diabetes & $190(31.7 \%)$ & $161(26.9 \%)$ & 0.066 & $306(38.2 \%)$ & $295(36.9 \%)$ & 0.570 \\
\hline COPD & $56(9.3 \%)$ & $34(5.7 \%)$ & 0.016 & $90(11.2 \%)$ & $86(10.7 \%)$ & 0.749 \\
\hline Hyperlipidemia & $286(47.7 \%)$ & $269(44.9 \%)$ & 0.325 & $380(47.5 \%)$ & $366(45.8 \%)$ & 0.483 \\
\hline Hemodialysis & $91(15.2 \%)$ & $79(13.1 \%)$ & 0.321 & $120(15.0 \%)$ & $110(13.8 \%)$ & 0.476 \\
\hline
\end{tabular}

and functional validation. Are they also associated with POAF? To validate the hypothesis, we conducted this study to evaluated the positive findings for POAF susceptibility after CABG among Chinese population.

\section{RESULTS}

\section{Clinical characteristics of the study participants}

Characteristics of the cohort, stratified by the occurrence of POAF, are shown in Table 1. There were no significant differences between the POAF cases and the non-POAF cases for the mean age or gender distribution. This suggested that the matching based on these two variables was adequate. People were generally comparable for medical history of hypertension, diabetes, hyperlipidemia, and hemodialysis. While POAF patients have higher BMI $(P=0.005)$, and more likely to be COPD patients $(P=0.016)$, compared with the non POAF patients in stage I.

\section{Genotype distribution of 6 polymorphisms in 5 novel loci}

Table 2 showed the genotypic frequencies of 6 polymorphisms in 5 novel loci (NEURL rs 12415501 and rs6584555, CAND2 rs4642101, GJA1 rs13216675, TBX5 rs 10507248, and CUX2 rs6490029) in POAF patients and non-POAF patients, together with their associations with POAF susceptibility after CABG among Chinese Han people. Four SNPs in 3 loci (NEURL rs12415501 and rs6584555, CAND2 rs4642101, and CUX2 rs6490029) were significantly associated with POAF susceptibility after $\mathrm{CABG}$ among Chinese Han people. For NEURL rs 12415501, each additional copy of minor allele A was associated with a 1.29-fold increased risk of developing POAF $(\mathrm{OR}=1.29,95 \% \mathrm{CI}$ : 1.03-1.60, $P=0.023)$ under the log-additive model. Compared with individuals with the GG genotype, the OR for developing POAF was 1.22 (95\% CI: 0.92-1.63)among those with the AG genotype, and 1.51 (95\% CI: 0.93-2.49) for those with the AA genotype. NEURL rs6584555 were also replicated in our study (per $\mathrm{C}$ allele: $\mathrm{OR}=1.30,95 \% \mathrm{CI}$ : 1.06-1.60, $P=$ 0.012). For CAND2 rs4642101, each additional copy of minor allele $\mathrm{G}$ was associated with a 1.30-fold increased risk of developing POAF (OR $=1.30,95 \% \mathrm{CI}$ : 1.06-1.55, $P=0.007)$ under the log-additive model. Compared with individuals with the TT genotype, the OR for developing POAF was 1.28 (95\% CI: 1.02-1.67) among those with the TG genotype, and 1.57 (95\% CI: 1.03-2.35) for those with the GG genotype. Another significant loci was CUX2 rs6490029, for which each additional copy of minor allele $\mathrm{G}$ was associated with a 0.77 -fold decreased risk of developing POAF $(\mathrm{OR}=0.77,95 \% \mathrm{CI}$ : 0.63-0.94, $P$ $=0.008)$ under the log-additive model. Compared with individuals with the AA genotype, the OR for developing POAF was 0.88 (95\% CI: 0.69-1.12) among those with the GA genotype, and 0.46 (95\% CI: 0.28-0.76) for those with the GG genotype.

\section{Replication of the 3 significant loci and functional validation}

To enhance the reliability of the findings, we replicated NEURL rs6584555, CAND2 rs4642101, and CUX2 rs6490029 in an independent stage II. As shown in Table 3, NEURL rs6584555 and CAND2 rs4642101 were found to be significantly associated with POAF susceptibility. When pooled together, for NEURL rs6584555, each additional copy of minor allele $C$ was associated with a 1.29 -fold increased risk of developing POAF $\left(\mathrm{OR}=1.29,95 \% \mathrm{CI}: 1.13-1.48, P=1.7 \times 10^{-4}\right)$ under the log-additive model. Compared with individuals with the TT genotype, the OR for developing POAF was 1.46 (95\% CI: 1.14-1.88)among those with the CC genotype; for CAND2 rs4642101, each additional copy of minor allele $\mathrm{G}$ was associated with a 1.21-fold increased risk of developing POAF $(\mathrm{OR}=1.21,95 \% \mathrm{CI}: 1.08-1.36, P=$ 
Table 2: Logistic regression analysis of genetic predictors of postoperative atrial fibrillation in the study populations of stage I

\begin{tabular}{|c|c|c|c|}
\hline & $\operatorname{POAF}(N=600)$ & No POAF $(N=600)$ & OR (95\% CIs) * \\
\hline \multicolumn{3}{|c|}{ NEURL rs12415501 } & \\
\hline GG & $420(70.0 \%)$ & $451(75.1 \%)$ & 1.00 (reference) \\
\hline GA & $139(23.2 \%)$ & $120(20.0 \%)$ & $1.22(0.92-1.63)$ \\
\hline $\mathrm{AA}$ & $41(6.8 \%)$ & $29(4.9 \%)$ & $1.51(0.93-2.49)$ \\
\hline A vs $G$ & & & $1.29(1.03-1.60)$ \\
\hline $\mathrm{P}$ trend & & & 0.023 \\
\hline \multicolumn{4}{|c|}{ NEURL rs6584555 } \\
\hline TT & $424(70.7 \%)$ & $452(75.3 \%)$ & 1.00 (reference) \\
\hline $\mathrm{TC}$ & $105(17.5 \%)$ & $97(16.1 \%)$ & $1.15(0.85-1.57)$ \\
\hline $\mathrm{CC}$ & $71(11.8 \%)$ & $51(8.6 \%)$ & $1.48(1.01-2.17)$ \\
\hline $\mathrm{C}$ vs T & & & $1.30(1.06-1.60)$ \\
\hline $\mathrm{P}$ trend & & & 0.012 \\
\hline \multicolumn{4}{|c|}{ CAND2 rs4642101 } \\
\hline TT & $285(47.5 \%)$ & $329(54.9 \%)$ & 1.00 (reference) \\
\hline TG & $250(41.7 \%)$ & $223(37.1 \%)$ & $1.28(1.02-1.67)$ \\
\hline GG & $65(10.8 \%)$ & $48(8.0 \%)$ & $1.57(1.03-2.35)$ \\
\hline G vs T & & & $1.30(1.06-1.55)$ \\
\hline $\mathrm{P}$ trend & & & 0.007 \\
\hline \multicolumn{4}{|c|}{ GJA1 rs13216675 } \\
\hline $\mathrm{TT}$ & $200(33.3 \%)$ & $194(32.3 \%)$ & 1.00 (reference) \\
\hline $\mathrm{TC}$ & $260(43.3 \%)$ & $241(40.1 \%)$ & $1.05(0.80-1.36)$ \\
\hline $\mathrm{CC}$ & $140(23.4 \%)$ & $165(28.6 \%)$ & $0.82(0.61-1.11)$ \\
\hline $\mathrm{C}$ vs T & & & $0.90(0.77-1.06)$ \\
\hline $\mathrm{P}$ trend & & & 0.204 \\
\hline \multicolumn{4}{|c|}{ TBX5 rs10507248 } \\
\hline GG & $305(50.8 \%)$ & $293(48.9 \%)$ & 1.00 (reference) \\
\hline GT & $209(34.9 \%)$ & $211(35.1 \%)$ & $0.95(0.74-1.22)$ \\
\hline TT & $86(14.3 \%)$ & $96(26.0 \%)$ & $0.86(0.62-1.20)$ \\
\hline T vs G & & & $0.92(0.78-1.09)$ \\
\hline $\mathrm{P}$ trend & & & 0.338 \\
\hline \multicolumn{4}{|c|}{ CUX2 rs6490029 } \\
\hline $\mathrm{AA}$ & $375(62.5 \%)$ & $344(57.3 \%)$ & 1.00 (reference) \\
\hline GA & $202(33.7 \%)$ & $211(35.1 \%)$ & $0.88(0.69-1.12)$ \\
\hline GG & $23(3.8 \%)$ & $46(7.6 \%)$ & $0.46(0.28-0.76)$ \\
\hline G vs A & & & $0.77(0.63-0.94)$ \\
\hline $\mathrm{P}$ trend & & & 0.008 \\
\hline
\end{tabular}

* OR was adjusted by Age, gender, BMI and COPD

$P$ value in bold means statistically significant.

$9.8 \times 10^{-4}$ ) under the log-additive model. Compared with individuals with the TT genotype, the OR for developing POAF was 1.24 (95\% CI: 1.06-1.45) among those with the TG genotype, and 1.38 (95\% CI: 1.06-1.79) for those with the GG genotype.

We then assessed the influence of NEURL rs6584555 and CAND2 rs4642101 on the expression of its corresponding genes in RAA samples. As shown in (Figure 1A and 1B), the AF risk allele of NEURL rs6584555 and CAND2 rs4642101 correlated with an increased expression of its corresponding genes $(P<$ $0.001)$.

\section{DISCUSSION}

Atrial fibrillation is a common arrhythmia with major public health implications due to its high prevalence, significant morbidity and considerable 
A

\section{rs6584555}

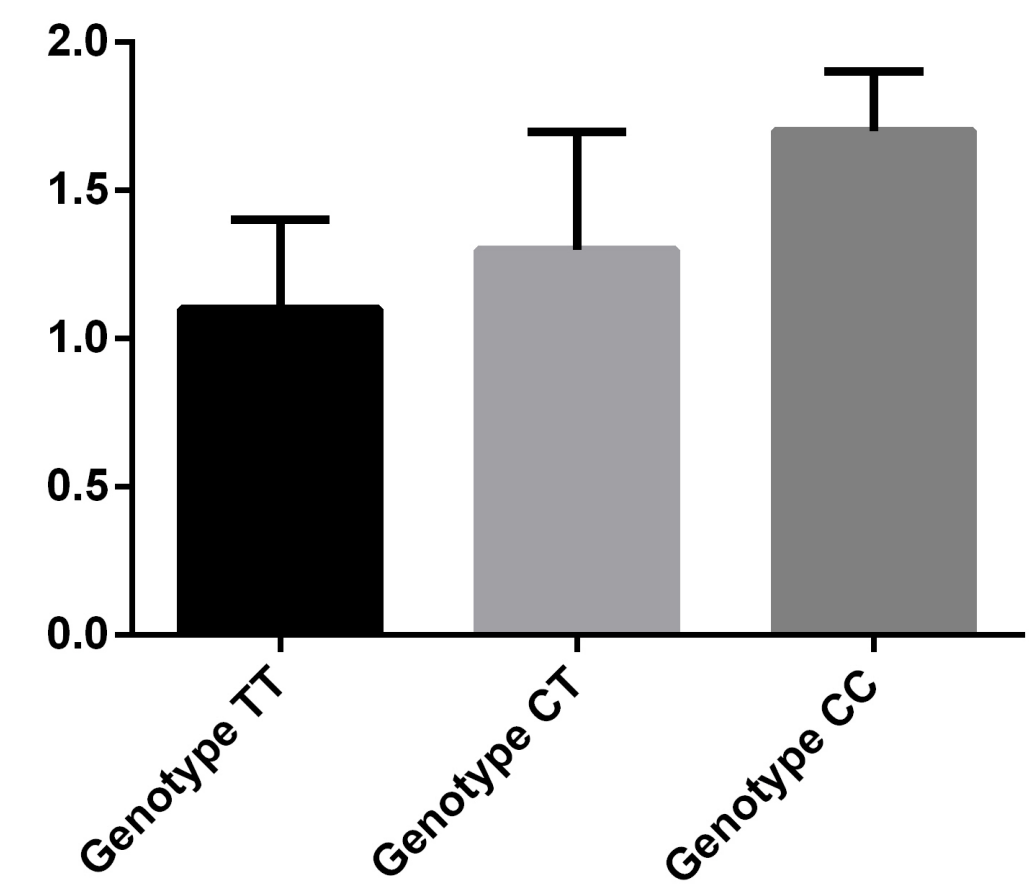

- Genotype TT Genotype CT Genotype CC

B

\section{rs4642101}

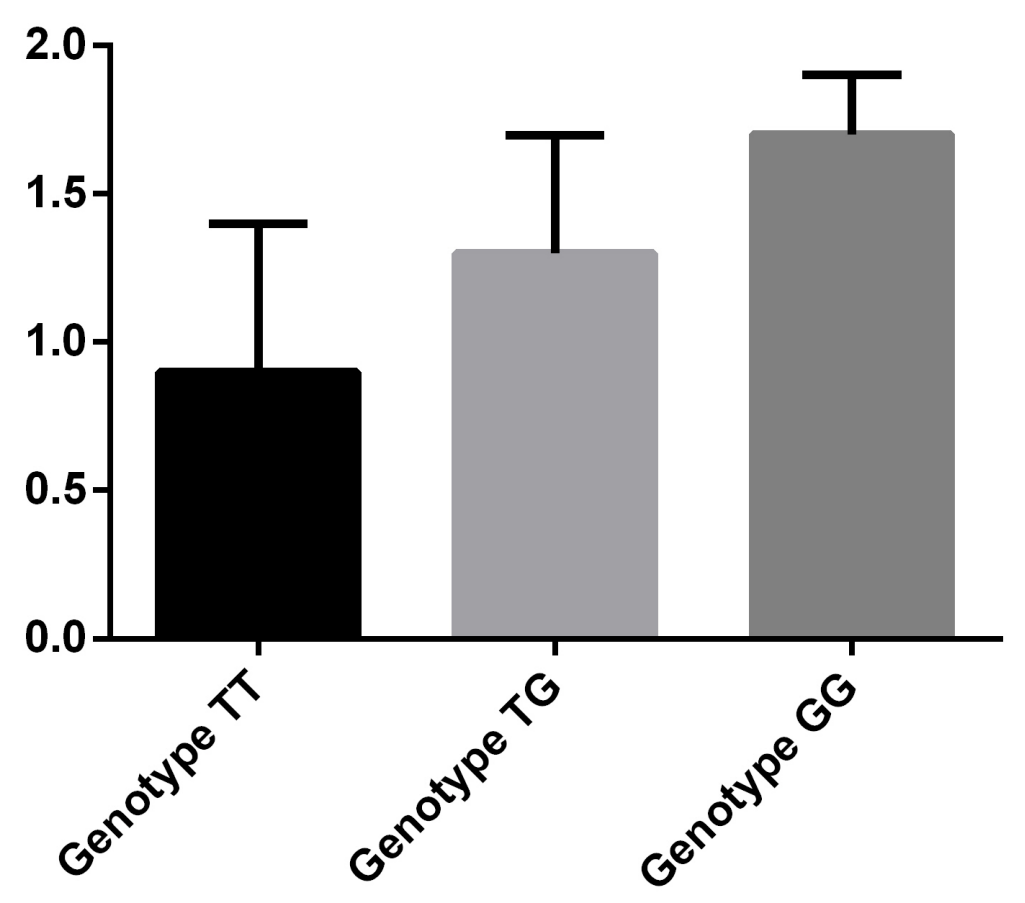

- Genotype TT - Genotype TG Genotype GG

Figure 1: Associations between expression of NEURL, CAND2 and its genetic variants. A. Associations between rs6584555 and expression of NEURL; B. Associations between rs4642101and expression of CAND2. 
Table 3: Logistic regression analysis of genetic predictors of postoperative atrial fibrillation in the study populations of stage II

\begin{tabular}{|c|c|c|c|c|c|c|}
\hline & \multicolumn{3}{|l|}{\begin{tabular}{|l|} 
Stage II \\
\end{tabular}} & \multicolumn{3}{|l|}{ Pooled results } \\
\hline & $\begin{array}{l}\text { POAF } \quad(N= \\
800)\end{array}$ & $\begin{array}{l}\text { No POAF }(N= \\
800)\end{array}$ & OR $(95 \%$ CIs $) *$ & $\begin{array}{l}\text { POAF } \quad(N= \\
\text { 1400) }\end{array}$ & $\begin{array}{l}\text { No POAF }(N= \\
1400)\end{array}$ & OR (95\% CIs) \\
\hline \multicolumn{7}{|c|}{ NEURL rs6584555 } \\
\hline TT & $568(71.0 \%)$ & $605(75.6 \%)$ & 1.00 (reference) & $992(70.8 \%)$ & $1057(75.5 \%)$ & 1.00 (reference) \\
\hline $\mathrm{TC}$ & $141(17.6 \%)$ & $128(16.0 \%)$ & $1.17(0.90-1.53)$ & $246(17.6 \%)$ & $225(16.1 \%)$ & $1.16(0.95-1.42)$ \\
\hline $\mathrm{CC}$ & $91(11.4 \%)$ & $67(8.4 \%)$ & $1.44(1.03-2.02)$ & $162(11.6 \%)$ & $118(8.4 \%)$ & $1.46(1.14-1.88)$ \\
\hline C vs T & & & $1.29(1.07-1.54)$ & & & $1.29(1.13-1.48)$ \\
\hline $\mathrm{P}$ trend & & & 0.005 & & & $1.7 \times 10^{-4}$ \\
\hline \multicolumn{7}{|c|}{ CAND2 rs4642101 } \\
\hline $\mathrm{TT}$ & $394(49.2 \%)$ & $(54.2 \%)^{434}$ & 1.00 (reference) & $679(48.6 \%)$ & $763(54.5 \%)$ & 1.00 (reference) \\
\hline TG & $322(40.2 \%)$ & $293(36.6 \%)$ & $1.21(0.98-1.49)$ & $572(40.8 \%)$ & $516(36.9 \%)$ & $1.24(1.06-1.45)$ \\
\hline GG & $84(10.6 \%)$ & $73(9.2 \%)$ & $1.26(0.90-1.78)$ & $149(10.6 \%)$ & $121(8.6 \%)$ & $1.38(1.06-1.79)$ \\
\hline G vs T & & & $1.17(1.00-1.36)$ & & & $1.21(1.08-1.36)$ \\
\hline P trend & & & 0.040 & & & $9.8 \times 10^{-4}$ \\
\hline \multicolumn{7}{|c|}{ CUX2 rs6490029 } \\
\hline $\mathrm{AA}$ & $472(58.9 \%)$ & $454(56.8 \%)$ & 1.00 (reference) & & & \\
\hline GA & $272(34.0 \%)$ & $280(35.0 \%)$ & $0.93(0.76-1.15)$ & & & \\
\hline GG & $56(7.1 \%)$ & $66(8.2 \%)$ & $0.82(0.56-1.19)$ & & & \\
\hline G vs A & & & $0.91(0.77-1.07)$ & & & \\
\hline P trend & & & 0.252 & & & \\
\hline
\end{tabular}

* OR was adjusted by Age, gender, BMI and COPD

$\mathrm{P}$ value in bold means statistically significant.

associated healthcare costs. There is strong evidence for heritability of POAF. In this large population-based, two-stage, nested case-control study, we identified 2 loci (NEURL rs6584555, and CAND2 rs4642101) were significantly associated with susceptibility of POAF after CABG. Testing for these genetic markers could improve risk stratification and potentially personalize therapy for preventing POAF.

The economic and clinical implications of postoperative $\mathrm{AF}$ are considerable. Many investigators have tried to identify predictors of AF after cardiac surgery for more than 2 decades. Although, the pathophysiology of $\mathrm{AF}$ has been extensively studied in the general population, it remains incompletely understood [22]. In 2003, Gaudino et al [23] identified that the $-174 \mathrm{G} / \mathrm{C}$ polymorphism of the promoter of the Interleukin- 6 gene has a role in the pathogenesis of postoperative atrial fibrillation. In current study, we identified that 2 loci (NEURL rs6584555, and CAND2 rs4642101) were significantly associated with increased risk of POAF after CABG. In the study of Sinner et al [21], the most significantly associated novel AF locus was also identified is intronic to the gene NEURL, which encodes an E3 ubiquitin ligase. The human NEURL has been recently determined and found to map to chromosome 10q25.1 within the region frequently deleted in malignant astrocytomas [24]. Using embryonic zebrafish, Sinner et al [21] found that knockdown of the NEURL orthologous specifically altered atrial action potential duration without affecting cardiac contractile function or heart rate. CAND2 gene encodes a TATA-binding protein, TIP120b, which is muscle-specific and critical for myogenesis, and the eQTL analyses also indicate that the risk allele is associated with increased expression of CAND2 [21]. Expression of CUX2 in postmitotic neurons contributes to the maintenance of genome integrity through its stimulation of oxidative DNA damage repair [25]. Interesting, CUX2 loci were also significantly associated with ischemic stroke [21]. In our functional validation experiments, we found the AF risk allele of NEURL rs6584555 and CAND2 rs4642101 correlated with an increased expression of its corresponding genes $(P<0.001)$. These also proved the previous findings.

Our study has several limitations. We studied the genetic polymorphisms in Chinese undergoing CABG; therefore, our findings cannot be generalized to other ethnicities. In addition, we did not validate our findings in another independent cohort since the limited sample size we enrolled. We also didn't evaluated the interaction 
between these polymorphisms and preoperative BB, statin, anti-arrhythmic use, as well as their associations with term development of AF and mortality in CABG patients have not been previously described and will need further validation. Third, we used an $\alpha$ level of 0.05 . Although the cohorts are likely underpowered for rigorous identification of associations by genome-wide genotyping techniques, surgical populations with detailed phenotype of AF or other adverse outcomes are rarely available. However, they examine important public health issues in an increasingly aged population.

Conclusively, NEURL rs6584555, and CAND2 rs4642101 are identified to be independently associated with risk of POAF. These findings delineate an important genetic role in the etiology of POAF and provide a detailed genomic landscape in which to examine biological mechanisms.

\section{MATERIALS AND METHODS}

\section{Subjects}

Patients undergoing primary CABG surgery without planned concurrent valve surgery were enrolled. The exclusion criteria were as follows: prior cardiac surgery; emergency surgical procedure; acute coronary syndrome; prior myocardial infarction; congestive heart failure; significant vascular heart disease; prior implantation of a permanent pacemaker, implantable cardioverter defibrillator, or cardiac resynchronization therapy defibrillator converted to a standard on-pump procedure; and use of class I or class III anti-arrhythmic agents. Also, the patients were excluded if they were not in sinus rhythm during echocardiography. POAF was defined as the occurrence of AF identified from nursing, physician, and perioperative ECG records, during the postoperative period of the primary hospitalization defined as the duration of contiguous hospitalization in the same institution as the surgery occurred. Finally, a total of 1,400 POAF patients and 1,400 non-POAF patients in two-stage were prospectively enrolled, respectively. An uniform questionnaire was used to collect patient demographics, preoperative and procedural factors, perioperative medication use, postoperative outcomes obtained from patient interview and medical records, and staff interviews. Right atrial appendage (RAA) samples of 200 individuals were randomly selected from the total patients with tissues available.. The study protocols were approved by appropriate institutional review boards, and participants were enrolled after informed written consent was obtained.

\section{DNA extraction and genotyping}

Genomic DNA was extracted from EDTAanticoagulated peripheral blood leukocytes by the saltingout method [26]. The genotyping were determined by using a polymerase chain reaction-restriction fragment length polymorphism method (PCR-RFLP). To confirm the genotyping results, PCR-amplified DNA samples were examined by DNA sequencing, and the results were $100 \%$ concordant.

\section{Quantification of gene expression using real-time PCR}

Expression of the candidate genes (NEURL and CAND2) in atrial tissues of 200 individuals who developed POAF was confirmed by real time PCR. POAF was present at the time of tissue acquisition Assays were performed using TaqMan gene expression probes and reagents (Life Technologies) and run on a 7900HT Real Time PCR System (Applied Biosystems). GAPDH was used as the reference gene.

\section{Statistical analyses}

Genotype and allele frequencies of the SNPs were compared between POAF cases and controls using the $\mathrm{x}^{2}$ test and Fisher's exact test when appropriate, and odds ratios (OR) and 95\% confidence intervals (CIs) were calculated to assess the relative risk conferred by a particular allele and genotype. For each of the SNPs, allelic associations with POAF were assessed using logistic regression analyses adjusted for the potential confounding factors. Demographic and clinical data between groups were compared by $\mathrm{x}^{2}$ test and by Student's-test. The linkage disequilibrium (LD) between the polymorphisms was quantified using the Shi's standardized coefficient D'. Statistical significance was assumed at the $P<0.05$ level. The SPSS statistical software package version 11.5 was used for all of the statistical analysis.

\section{ACKNOWLEDGMENTS}

Many thanks to the project team.

\section{CONFLICTS OF INTEREST}

The authors declare that there is no conflict of interest.

\section{REFERENCES}

1. Shirzad M, Karimi A, Tazik M, Aramin H, Hossein Ahmadi S, Davoodi S and Marzban M. Determinants of 
postoperative atrial fibrillation and associated resource utilization in cardiac surgery. Rev Esp Cardiol. 2010; 63:1054-1060.

2. Bramer S, van Straten AH, Soliman Hamad MA, Berreklouw E, van den Broek KC and Maessen JG. Body mass index predicts new-onset atrial fibrillation after cardiac surgery. Eur J Cardiothorac Surg. 2011; 40:11851190.

3. Silva RG, Lima GG, Guerra N, Bigolin AV and Petersen LC. Risk index proposal to predict atrial fibrillation after cardiac surgery. Revista brasileira de cirurgia cardiovascular : orgao oficial da Sociedade Brasileira de Cirurgia Cardiovascular. 2010; 25:183-189.

4. Mathew JP, Fontes ML, Tudor IC, Ramsay J, Duke P, Mazer CD, Barash PG, Hsu PH and Mangano DT. A multicenter risk index for atrial fibrillation after cardiac surgery. Jama. 2004; 291:1720-1729.

5. Banach M, Kourliouros A, Reinhart KM, Benussi S, Mikhailidis DP, Jahangiri M, Baker WL, Galanti A, Rysz J, Camm JA, White CM and Alfieri O. Postoperative atrial fibrillation - what do we really know? Curr Vasc Pharmacol. 2010; 8:553-572.

6. Almassi GH, Shroyer AL, Collins JF and Grover FL. ROOBY trial data demonstrates revascularization had no impact on POAF. Future cardiology. 2014; 10:157.

7. Bell DS and O'Keefe JH. Metabolic syndrome and postoperative atrial fibrillation (POAF). Eur Heart J. 2009; 30:1167-1168.

8. Mariscalco G, Klersy C, Zanobini M, Banach M, Ferrarese S, Borsani P, Cantore C, Biglioli P and Sala A. Atrial fibrillation after isolated coronary surgery affects late survival. Circulation. 2008; 118:1612-1618.

9. Worden JC and Asare K. Postoperative atrial fibrillation: role of inflammatory biomarkers and use of colchicine for its prevention. Pharmacotherapy. 2014; 34:1167-1173.

10. Varma PK. Prediction of postoperative atrial fibrillation after cardiac surgery: light at the end of the tunnel? Annals of cardiac anaesthesia. 2014; 17:187-190.

11. Danelich IM, Lose JM, Wright SS, Asirvatham SJ, Ballinger BA, Larson DW and Lovely JK. Practical management of postoperative atrial fibrillation after noncardiac surgery. J Am Coll Surg. 2014; 219:831-841.

12. Bohatch Junior MS, Matkovski PD, Giovanni FJ, Fenili R, Varella EL and Dietrich A. Incidence of postoperative atrial fibrillation in patients undergoing on-pump and offpump coronary artery bypass grafting. Revista brasileira de cirurgia cardiovascula. 2015; 30:316-324.

13. Mariscalco G, Biancari F, Zanobini M, Cottini M, Piffaretti G, Saccocci M, Banach M, Beghi C and Angelini GD. Bedside tool for predicting the risk of postoperative atrial fibrillation after cardiac surgery: the POAF score. Journal of the American Heart Association. 2014; 3:e000752.

14. Jiang MH, Su YM, Tang JZ, Shen YB, Deng XT, Yuan
DS, Wu J, Pan M and Huang ZW. Angiotensin-converting enzyme gene $2350 \mathrm{G} / \mathrm{A}$ polymorphism and susceptibility to atrial fibrillation in Han Chinese patients with essential hypertension. Clinics (Sao Paulo). 2013; 68:1428-1432.

15. Darbar D. Atrial Fibrillation Susceptibility Alleles on Chromosome 4q25 Modulate Response to Catheter Ablation. Journal of atrial fibrillation. 2010; 1.

16. Lubitz SA, Sinner MF, Lunetta KL, Makino S, Pfeufer A, Rahman R, Veltman CE, Barnard J, Bis JC, Danik SP, Sonni A, Shea MA, Del Monte F, Perz S, Muller M, Peters A, et al. Independent susceptibility markers for atrial fibrillation on chromosome 4q25. Circulation. 2010; 122:976-984.

17. Lemmens R, Buysschaert I, Geelen V, Fernandez-Cadenas I, Montaner J, Schmidt H, Schmidt R, Attia J, Maguire J, Levi C, Jood K, Blomstrand C, Jern C, Wnuk M, Slowik A, Lambrechts D, et al. The association of the $4 \mathrm{q} 25$ susceptibility variant for atrial fibrillation with stroke is limited to stroke of cardioembolic etiology. Stroke. 2010; 41:1850-1857.

18. Gai X, Zhang Z, Liang Y, Chen Z, Yang X, Hou J, Lan $\mathrm{X}$, Zheng $\mathrm{W}$ and Huang M. MMP-2 and TIMP-2 gene polymorphisms and susceptibility to atrial fibrillation in Chinese Han patients with hypertensive heart disease. Clin Chim Acta. 2010; 411:719-724.

19. Giusti B, Gori AM, Marcucci R, Sestini I, Saracini C, Sticchi E, Gensini F, Fatini C, Abbate R and Gensini GF. Role of C677T and A1298C MTHFR, A2756G MTR and $-786 \mathrm{C} / \mathrm{T}$ eNOS gene polymorphisms in atrial fibrillation susceptibility. PLoS One. 2007; 2:e495.

20. Liu L, Zhang L, Liu M, Zhang Y, Han X and Zhang Z. GRK5 polymorphisms and Postoperative Atrial Fibrillation following Coronary Artery Bypass Graft Surgery. Scientific reports. 2015; 5:12768.

21. Sinner MF, Tucker NR, Lunetta KL, Ozaki K, Smith JG, Trompet S, Bis JC, Lin H, Chung MK, Nielsen JB, Lubitz SA, Krijthe BP, Magnani JW, Ye J, Gollob MH, Tsunoda $\mathrm{T}$, et al. Integrating genetic, transcriptional, and functional analyses to identify 5 novel genes for atrial fibrillation. Circulation. 2014; 130:1225-1235.

22. Chua SK, Shyu KG and Lo HM. Postoperative atrial fibrillation and cardiac surgery. Circ J. 2014; 78:265.

23. Gaudino M, Andreotti F, Zamparelli R, Di Castelnuovo A, Nasso G, Burzotta F, Iacoviello L, Donati MB, Schiavello R, Maseri A and Possati G. The $-174 \mathrm{G} / \mathrm{C}$ interleukin-6 polymorphism influences postoperative interleukin-6 levels and postoperative atrial fibrillation. Is atrial fibrillation an inflammatory complication? Circulation. 2003; 108 Suppl 1:II195-199.

24. Pavlopoulos E, Kokkinaki M, Koutelou E, Mitsiadis TA, Prinos P, Delidakis C, Kilpatrick MW, Tsipouras P and Moschonas NK. Cloning, chromosomal organization and expression analysis of Neurl, the mouse homolog of Drosophila melanogaster neuralized gene. Biochim Biophys 
Acta. 2002; 1574:375-382.

25. Pal R, Ramdzan ZM, Kaur S, Duquette PM, Marcotte R, Leduy L, Davoudi S, Lamarche-Vane N, Iulianella A and Nepveu A. CUX2 Functions As an Accessory Factor in the Repair of Oxidative DNA Damage. J Biol Chem. 2015; 290:22520-22531.

26. Miller SA, Dykes DD and Polesky HF. A simple salting out procedure for extracting DNA from human nucleated cells. Nucleic Acids Res. 1988; 16:1215. 